\title{
Arbeitszeitrechtsgesetz - ArbZRG in der Fassung vom 6. Juni 1994 ArbZRG in der Fassung ArbZRG
}

\author{
Gesetz zur Vereinheitlichung und Flexibilisierung des Arbeitszeitrechts - \\ Auswirkungen auf den betrieblichen Ablauf, Erfahrungen nach einem Jahr
}

\author{
W. Werner
}

Auswirkungen auf den Funktionsbereich an allen Kliniken durch das Arbeitszeitrechtsgesetz in der Fassung vom 6.Juni 1994, dessen Umsetzung in unserem Hause am 1.1.1997 erfolgt. Ich möchte zuerst auf die rechtlichen Grundlagen hinweisen, dann auf die Auswirkungen an unserer Klinik mit 700 operativ zu versorgenden Betten hinweisen.

Grundlage für das Arbeitszeitgesetz ist für den Bereich der Krankenhäuser die Fassung vom 6. Juni 1994. Veröffentlicht im Bundesgesetzblatt I/ab Seite 1170.

Für den Funktionsdienst sind folgende $\S \S$ maßgebend $\S \S 1,3,4,5$ hieraus vor allem Absatz 5 in der Erläuterung und Anlage III der Übersicht. Hieraus besonders III/1, ab Position 30 bis 71, sowie der BAT, hieraus SR 2a.

\section{Die gesetzlichen Grundlagen des ArbZG für Anwendung im Krankenhaus}

\section{$\S 1$ des ArbZG besagt: Zweck des Gesetzes}

1. Die Sicherheit und den Gesundheitsschutz der Arbeitnehmer bei der Arbeitszeitgestaltung und die Rahmenbedingungen für flexible Arbeitszeiten zu verbessern sowie

2. den Sonntag und die staatlich anerkannten Feiertage als Tage der Arbeitsruhe und der seelischen Erhebung der Arbeitnehmer zu schützen.

Dies bedeutet im Rahmen der Bereitschaftsregelung jedoch nichts anderes, als dass die Tage der Arbeitsruhe - sprich Sonn- und Feiertage - die meistgefragten Bereitschaftsdiensttage sind.

OP-JOURNAL 2000; 16: 206-209

(C) Georg Thieme Verlag Stuttgart · New York
Zweiter Abschnitt, §3: Arbeitszeit der Arbeitnehmer

Die werktägliche Arbeitszeit der Arbeitnehmer darf 8 Stunden nicht überschreiten. Sie kann auf bis zu 10 Stunden nur verlängert werden, wenn innerhalb von 6 Kalendermonaten oder innerhalb von 24 Wochen im Durchschnitt 8 Stunden werktäglich nicht überschritten werden.

\section{$\S 4$ Ruhepausen}

Die Arbeit ist durch im voraus feststehende Ruhepausen von mindestens 30 Minuten bei einer Arbeitszeit von mehr als 6 Stunden bis zu 9 Stunden und 45 Minuten bei einer Arbeitszeit von mehr als 9 Stunden insgesamt zu unterbrechen. Die Ruhepausen nach Satz 1 können in Zeitabschnitte von jeweils mindestens 15 Minuten aufgeteilt werden.

Länger als 6 Stunden hintereinander dürfen Arbeitnehmer nicht ohne Ruhepause beschäftigt werden.

\section{$\S 5$ Ruhezeiten}

1. Die Arbeitnehmer müssen nach Beendigung der täglichen Arbeitszeit eine ununterbrochene Ruhezeit von mindestens 11 Stunden haben.

2. Die Dauer der Ruhezeit des Absatzes 1 kann in Krankenhäusern und anderen Einrichtungen zur Behandlung, Pflege und Betreuung von Personen, in Gaststätten und anderen Einrichtungen zur Bewirtung und Beherbergung, in Verkehrsbetrieben, beim Rundfunk sowie in der Landwirtschaft und in der Tierhaltung um bis zu eine Stunde verkürzt werden, wenn jede Verkürzung der Ruhezeit innerhalb eines Kalendermonats oder innerhalb von 4 Wochen durch Verlängerung einer anderen Ruhezeit auf mindestens 12 Stunden ausgeglichen wird.

3. Abweichend von Absatz 1 können in Krankenhäusern und anderen Einrichtungen zur Behandlung, Pflege und Be- treuung von Personen Kürzungen der Ruhezeit durch Inanspruchnahmen während des Bereitschaftsdienstes oder der Rufbereitschaft, die nicht mehr als die Hälfte der Ruhezeit betragen, zu anderen Zeiten ausgeglichen werden.

Nur sollte man sich fragen: Wann?

Erläuterung zu Absatz 3:

Durch Absatz 3 wird sichergestellt, dass in Krankenhäusern beschäftigte Personen (z.B. Ärzte und Krankenschwestern) trotz Arbeitsleistung während des Bereitschaftsdienstes oder der Rufbereitschaft planmäßig im Anschluss an diese Dienste ihre Tätigkeit aufnehmen können, ohne dass im Anschluss an Arbeitsleistungen während dieser Dienste eine erneute 10-stündige Ruhezeit erforderlich wird. Arbeitszeiten während des Bereitschaftsdienstes oder der Rufbereitschaft können daher $\mathrm{zu}$ anderen Zeiten ausgeglichen werden. Ein Zeitraum, innerhalb dessen der Ausgleich zu erfolgen hat, wird gesetzlich nicht festgelegt. Der Ausgleich kann auch während anderer Bereitschaftsdienste oder Rufbereitschaften erfolgen, soweit keine Arbeitsleistung während dieser Dienste erbracht wird.

\section{Folgen der Unterbrechung}

Wird die Ruhezeit durch eine Arbeitsleistung unterbrochen, z.B. im Rahmen eines Bereitschaftsdienstes oder einer Rufbereitschaft, und soll die normale Arbeitstätigkeit planmäßig vor Ablauf der neu zu laufen beginnenden mindestens 11-stündigen Ruhezeit anfangen, so ist der Arbeitsbeginn entsprechend zu verschieben. Der Arbeitnehmer kann für die hierdurch ggf. ausfallende Arbeitszeit keine Bezahlung verlangen, wenn nicht 8 oder einzelvertraglich etwas anderes vereinbart ist (vgl. BAG vom 5.7.1976 AP Nr.10 zu §12 AZO). 
Es handelt sich um den Fall einer von keiner Seite zu vertretenden Unmöglichkeit, bei der nach $\S 323$ Absatz 1 BGB der Anspruch auf die Gegenleistung (Vergütung) entfällt.

\section{Ausnahmen für Bereitschaftsdienste und Rufbereitschaften in Krankenhäusern (Absatz 3)}

\section{Problemstellung}

Nach bisherigem Recht mussten weder Ärzte noch das Pflegepersonal in Krankenhäusern aufgrund öffentlich-rechtlicher Schutzvorschriften eine ununterbrochene Ruhezeit von 11 Stunden im Anschluss an die tägliche Arbeitszeit haben. Die durch das Arbeitszeitrechtsgesetz aufgehobene Verordnung über die $\mathrm{Ar}$ beitszeit in Krankenpflegeanstalten vom 13.2.1924, die die Arbeitszeit des Pflegepersonals regelte, enthielt keine Ruhezeitenregelung. Nach der Rechtsprechung des BAG fanden auf angestellte Ärzte in Krankenhäusern weder die Bestimmungen der Verordnung über die Arbeitszeit in Krankenpflegeanstalten noch die Bestimmungen der Arbeitszeitordnung Anwendung (BAG vom 8.11.1962 AP Nr. 25 zu §611 BGB Ärzte, Gehaltsansprüche).

Das Arbeitszeitgesetz dehnt den öffentlich-rechtlichen Arbeitsschutz einheitlich auf alle Arbeitnehmer, also auch auf die angestellten Krankenhausärzte und das Krankenpflegepersonal, aus. Damit werden für die im Krankenhausbereich beschäftigten Arbeitnehmer erstmals Mindestruhezeiten nach Beendigung der täglichen Arbeitszeit festgeschrieben.

Im Krankenhausbereich ist es üblich, dass im Anschluss an die tägliche Arbeitszeit von Ärzten und dem Pflegepersonal Bereitschaftsdienste geleistet werden. So ist z.B. folgendes Arbeitszeitmodell in Krankenhäusern verbreitet:

- 8 Stunden Arbeitszeit

- 16 Stunden Bereitschaftsdienst

- 8 Stunden Arbeitszeit

Für die Arbeitsverhältnisse in Krankenhäusern gilt i.d.R. der BAT. Dieser unterscheidet in der Sonderregelung für Ärzte in Krankenhäusern (SR 2c BAT) vier Formen des Bereitschaftsdienstes, die nach der Arbeitsleistung innerhalb des Bereitschaftsdienstes klassifiziert werden: Die Basisgrundvergütung, für den angeordneten Bereitschaftsdienst beträgt in der Regel 25\% einer Überstundenvergütung, nur in der Bereitschaftsdienststufe „A“, sind dies $10 \%$.
Stufe A 0-10 von Hundert Arbeitsleistung innerhalb des Bereitschaftsdienstes

Stufe B 10-25 von Hundert Arbeitsleistung innerhalb des Bereitschaftsdienstes

Stufe C 25-40 von Hundert Arbeitsleistung innerhalb des Bereitschaftsdienstes

Stufe D 40-49 von Hundert Arbeitsleistung innerhalb des Bereitschaftsdienstes

Die Inanspruchnahmen während des Bereitschaftsdienstes oder der Rufbereitschaft sind auf die tägliche Arbeitszeit anzurechnen. Die Höchstarbeitszeitgrenzen des $\S 3$ und $\S 6$ Absatz 2 dürfen nicht überschritten werden. Hat der Arbeitnehmer bereits bis zur Grenze der höchstzulässigen Arbeitszeit - d.h. 10 Stunden gearbeitet, darf er während des Bereitschaftsdienstes bzw. der Rufbereitschaft nicht mehr zu Arbeitsleistungen herangezogen werden, so dass sich in diesem Fall die Anordnung der Dienste verbietet. Die Tarifvertragsparteien und ggf. die Betriebspartner können allerdings nach $\S 7$ Absatz 2 Nr. 3 etwas anderes vereinbaren.

Um insbesondere kleinen Krankenhäusern die organisatorische Umstellung auf diese Neuregelung zu ermöglichen, enthält §26 eine Übergangsregelung, nach der die Ruhezeitenregelung des $\S 5$ für Ärzte und das Pflegepersonal in Krankenhäusern erst ab 1.1.1996 anzuwenden ist.

Hier tritt dann die Änderung aus der Ergänzung vom September 1995 in Kraft, die besagt, dass die Ruhezeitenregelung für Ärzte und Pflegekräfte erst zum 01. 01.1997 in Kraft treten muss.

Abweichungsmöglichkeiten, Ausnahmen, Mitbestimmung und Bußgeldtatbestand

Die Tarifvertragsparteien und ggf. Betriebspartner können nach $\S 7$ Absatz 1 , Nr.3 und $\S 7$ Absatz 2 Nr.1 - 4 von $\S 5$ Absatz 1 abweichende Regelungen treffen. In den außergewöhnlichen Fällen des §14 kann Kraft Gesetzes von der Ruhezeitenregelung des $\S 5$ und ggf. den Regelungen nach $\S 7$ abgewichen werden. Ferner kann die Aufsichtsbehörde in den Fällen des $\S 15$ Absatz 1 Nr. 3 und 4 abweichende Ruhezeitenregelungen bewilligen.

Die Betriebs- und Personalräte haben bezüglich der Ruhezeitenregelung kein zwingendes Mitbestimmungsrecht. Sie können Lage und Dauer der Ruhezeit al- lerdings im Rahmen ihres Mitbestimmungsrechts über Beginn und Ende der täglichen Arbeitszeit einschließlich der Pausen sowie Verteilung der Arbeitszeit auf die einzelnen Wochentage beeinflussen (hier auch $\S 2$ Randnummer $15 \mathrm{ff}$.).

Nach §22 Absatz 1 Nr.3 handelt ordnungswidrig, wer als Arbeitgeber vorsätzlich oder fahrlässig entgegen $\S 5 \mathrm{Ab}$ satz 1 die Mindestruhezeit nicht gewährt oder entgegen $\$ 5$ Absatz 2 die Verkürzung der Ruhezeit durch Verlängerung einer anderen Ruhezeit nicht oder nicht rechtzeitig ausgleicht.

Das Krankenhaus darf nach den tarifvertraglichen Bestimmungen bei seiner $\mathrm{Ar}$ beitszeitgestaltung keine Bereitschaftsdienste vorsehen, wenn zu erwarten ist, dass die Arbeitsleistung mehr als 49 von Hundert der Zeit des Bereitschaftsdienstes beträgt.

Im Kalendermonat dürfen grundsätzlich in den Stufen A und B nicht mehr als 7, in den Stufen $C$ und D nicht mehr als 6 Bereitschaftsdienste angeordnet werden, jeder weitere Bereitschaftsdienst wird in Stufe C mit 80\%, in Stufe D mit 100\% vergütet.

Die Regelungen zur Ruhezeit sehen wie folgt aus: Wird der Arzt oder die Pflegeperson an einem Kalendertag, an dem er eine Arbeitszeit von mindestens 7,5 Stunden abgeleistet hat, zu einem Bereitschaftsdienst der Stufe C oder D herangezogen, der mindestens 12 Stunden dauert, muss ihm nach diesem Bereitschaftsdienst eine Ruhezeit von mindestens 8 Stunden gewährt werden.

Durch die Schaffung des Arbeitszeitrechtsgesetzes vom 6. Juni 1994 wurde dem Wohle des einzelnen Mitarbeiters Rechnung getragen. Bedeutete es bis zu seiner Einführung und Umsetzung einen anstrengenden Dienst, auch und insbesondere nach geleistetem Bereitschaftsdienst, so muss man mit der jetzigen wirtschaftlichen Situation erst einmal fertig werden. Natürlich steht die Freizeit und die Ruhepause nicht erst seit der Einführung des Gesetzes im Vordergrund, doch sollte niemand vergessen, in wie weit sich die wirtschaftlichen Aspekte doch nun durch die Geldbörse der Mitabeiter ziehen.

Die im Funktionsdienst tätigen Mitarbeiter, hier insbesondere die Krankenschwestern und Krankenpfleger, ob mit oder ohne Fachausbildung, werden im Rah- 
men des Bereitschaftsdienstes der Stufen $C$ und $D$ doch derart bestraft, dass sie, trotz einer Auslastung von über $40 \%$ im Durchschnitt, am folgenden Tag mit einem freien Tag, unter Abzug von 7,7 Stunden aus dem berechenbaren Überstundenaufkommen, belastet werden. Jede Kollegin und jeder Kollege muss diesen Freizeitausgleich in Anspruch nehmen, da eine Verrechnung mit Überstunden nicht möglich ist.

Für unsere Klinik ergaben sich aus der Fassung des Arbeitszeitrechtsgesetz gravierende, dienstliche Änderungen.

Bei 5 Mitarbeitern, die am Bereitschaftsdienst täglich teilnehmen, kam es zu einem wöchentlichen Stundenausfall von 192,5 Stunden, zusätzlich zu dem bedingt durch Urlaub bzw. Krankheit hervorgerufenen Ausfall. Bedingt durch die Deckelung und der damit verbundenen Einschnitte im Personalwesen wurde eine Mittelfreigabe jedoch nicht für die gesamte Stundenzahl plus Ausfallzeiten gewährt, es wurden nur 4 Neueinstellungen genehmigt. Dadurch wird die personelle Situation gerade nicht verbessert. Die 5 bereitschaftsdienstleistenden Mitarbeiter kommen aus zwei Abteilungen: 2 Mitarbeiter aus der Anästhesie und 3 Mitarbeiter aus der Operationsabteilung.

Hiermit werden im Bereitschaftsdienst 8 Fachbereiche operativ verorgt. Die vier neuen Mitarbeiter wurden aufgeteilt in 1,5 Mitarbeiter für den Bereich Anästhesie und 2,5 Mitarbeiter für die Operationsabteilung. Dass es sich hierbei um eine recht schwierige Situation handelt, muss ich nicht gesondert herausstellen. Im gesamten Klinikum gilt für den Funktionsdienst eine tägliche Stammarbeitszeit von $7.18 \mathrm{Uhr}$ bis $15.30 \mathrm{Uhr}$, mit einer Pause von 30 Minuten, daraus errechnet sich die 38,5-Stunden-Woche.

Im Bereich des Pflegepersonals, sowohl für den Anästhesie- als auch den OP-Bereich, gibt es einen Zwischendienst, der täglich wechselt und von Montag bis Freitag durchgeführt werden muss. Hierbei ist der Arbeitsablauf generell festgelegt, kann aber nur selten eingehalten werden, da oft zwei oder drei Säle in den Dienst gehen (Kommentar der Operateure: „Das Team geht am nächsten Tag sowieso heim!“). Die tägliche Arbeitszeit vom Zwischendienst beginnt um $11.03 \mathrm{Uhr}$ und endet um 19.15 Uhr.

In den genannten Abteilungen konnte mit dem Personalschlüssel, der bis zum
31. Dezember 1996, auch mit wenig Personalfluktuation, Bestand hatte, bedingt durch die recht geringe Ausfallquote von $3-4,5 \%$ bei Krankheit und Kur gut gearbeitet werden. Mit der Einführung des Arbeitszeitrechtsgesetzes wurde die Rate der Ausfälle erheblich höher. Sie lag im Jahr 1997 bei durchschnittlich 8,5\% in den Fachbereichen Anästhesie und Op. Trotz oder gerade wegen der Personalverschiebung konnte erneut die Zahl der Eingriffe gesteigert werden. Im gesamten Funktionsbereich betrug die Steigerung circa 6\%, im Vergleich zum Vorjahr.

Im Rahmen des Bereitschaftsdienstes hat die Auslastung zugenommen. Hier insbesondere Punkte vom Tagungsprogramm, die dann in den Dienst gehen.

Die Auslastung im Bereitschaftsdienst stieg gering, jedoch das Überstundenaufkommen erheblich!

Jede Abteilung hat eine eigene Bereichsleitung, die aber im Tagesprogramm und im Bereitschafts- und Rufbereitschaftsdienst voll mitarbeitet.

Für den Pflegebereich Anästhesie und OP wird der Bereitschaftsdienst nach dem BAT mit der Stufe D vergütet.

Die Auslastung im Bereitschaftsdienst an unserer Klinik, liegt zwischen $37 \%$ und $50 \%$. Das bedeutet, dass der Durchschnitt der zu Grunde gelegten, dokumentierten Zeit bei 45,2\%, im Jahr 1997 lag.

Im Fachbereich Anästhesie ist die Auslastung erheblich, denn bei einem Großteil der Einsätze während des Bereitschaftsdienstes wird nur eine der zwei Bereitschaftsdienstleistenden benötigt. Die erhebliche Auslastung wird von den Einsätzen in den Außenkliniken verursacht:

$$
\begin{aligned}
& \text { Ortho-Op } \\
& \text { Endoskopie, hier insbesondere eine } \\
& \text { große Urologie } \\
& \text { Augen-Op } \\
& \text { CT } \\
& \text { Chirurg. Eil- und Unfallambulanz } \\
& \text { LHKM } \\
& \text { Notfälle auf den Stationen }
\end{aligned}
$$

Auch das „Sommerloch“ konnte die durchschnittliche Auslastung erheblich senken.

Der Bereitschafts- und Rufbereitschaftsdienst beginnt um 15.30 Uhr und endet um 7.40 Uhr, dies von Montag bis Don- nerstag; freitags und an Vorfeiertagen von 15.30 Uhr bis $8.30 \mathrm{Uhr}$, samstags von 8.00 Uhr bis 8.30 Uhr und Sonntags, sowie an Feiertagen von 8.00 Uhr bis 8.00 Uhr. Somit besteht an Werktagen eine Übergabezeit von 22 Minuten, an Samstagen und Feiertagen von $30 \mathrm{Mi}-$ nuten und nach dem Sonntagsdienst von 42 Minuten.

Der Rufbereitschaftsdienst endet allerdings um 7.18 Uhr, mit dem Beginn der Stammarbeitszeit.

Die Dienste am Freitag, Vorfeiertag und Samstag werden nach dem BAT voll vergütet, mit der Berechnungsgrundlage $55 \%$ plus $25 \%$ für den angeordneten Hausdienst. Daraus ergeben sich die 80\% einer Überstundenvergütung. Die Dienste an den Werktagen werden nach dem BAT folgendermaßen abgerechnet: Ermittlung der berechenbaren Überstunden minus 7,7 Stunden bei Vollkräften, 5,75 Stunden bei einer Anstellung von $75 \%$ und 3,85 Stunden bei 50\%-Anstellung.

\section{Somit kommt es zu in Tab. 1 beschriebener Vergütung}

Daraus ergeben sich aus Tab. 2 zu entnehmenden finanziellen Einbußen für den gesamten Pflegebereich.

Somit lässt sich unschwer erkennen, dass es sich hier um erhebliche Einbußen in der Bruttovergütung handelt. Bei einem monatlichen Bereitschaftsdienstdurchschnitt kommt es zu Gehaltseinbußen von bis zu 7000,- DM brutto pro Jahr. Es kann sich jeder vorstellen, dass diese Situation nicht gerade förderlich ist.

Eine komplette Umstellung von Bereitschaftsdienst auf einen Schichtdienst ist aus der derzeitigen wirtschaftlichen Situation ebenfalls nicht möglich. Ich verweise gerne auf die utopische Zahl von DM 76500,-. Da an den Städtischen Kliniken Frankfurt am Main-Höchst das Pflegemodell in allen Abteilungen eingesetzt wird, wäre eine weitere Neueinstellung nicht möglich. Dies hätte zur Folge, dass im Zentral-OP 1 täglich im Frühdienst von 8 möglichen Operationssälen nur 5 betrieben werden könnten, im Spätdienst 3 OP-Säle, im Nachtdienst 2. Nur setzt dies eine komplette Umstrukturierung der zentralen Funktionsabteilungen in allen Fachbereichen voraus. Inwieweit der ärztliche Sektor bei einer solchen gravierenden Änderung mitziehen würde, ist bei der Anzahl der 
Tab. 1 Vergütungsberechnung bei Bereitschaftsdiensten

\begin{tabular}{lll} 
Mo-Do & $\begin{array}{l}15.30 \mathrm{Uhr} \text { bis } 7.40 \mathrm{Uhr}=16,10 \mathrm{Std} .=970 \mathrm{~min} \\
80 \%=776 \mathrm{~min}-462 \mathrm{~min}\end{array}$ & $314 \mathrm{~min}$ \\
\hline Freitag u. & $\begin{array}{l}15.30 \mathrm{Uhr} \text { bis } 8.30 \mathrm{Uhr}=17 \mathrm{Std} .=1020 \mathrm{~min}, \\
\text { Vorfeiertage }\end{array}$ & $816 \mathrm{~min}$ \\
\hline Samstag & $\begin{array}{l}8.00 \mathrm{Uhr} \text { bis } 8.30 \mathrm{Uhr}=24,30 \mathrm{Std} .=1470 \mathrm{~min}, 80 \% \\
=1176 \mathrm{~min} .\end{array}$ & \\
\hline Sonntag & $\begin{array}{l}8.00 \mathrm{Uhr} \text { bis } 8.00 \mathrm{Uhr}=24.00 \mathrm{Std} .=1440 \mathrm{~min}, 80 \% \\
=1152 \mathrm{~min} .-462 \mathrm{~min} .=\end{array}$ & \\
& &
\end{tabular}

\begin{tabular}{|c|c|c|c|c|}
\hline Tage & $\begin{array}{l}\text { Vergütungs- } \\
\text { gruppe }\end{array}$ & bis 31.12 .96 & ab 1.1.98 & $\begin{array}{l}\text { Std.-Lohn } \\
\text { brutto }\end{array}$ \\
\hline \multirow[t]{4}{*}{ Montag-Donnerstag } & KR V & $355,45 \mathrm{DM}$ & $147,89 \mathrm{DM}$ & 9,18 DM \\
\hline & KR Va & $365,14 \mathrm{DM}$ & $151,98 \mathrm{DM}$ & 9,44 DM \\
\hline & KR VI & $379,24 \mathrm{DM}$ & $157,94 \mathrm{DM}$ & 9,81 DM \\
\hline & KR VII & $390,87 \mathrm{DM}$ & $162,65 \mathrm{DM}$ & $10,10 \mathrm{DM}$ \\
\hline \multirow[t]{4}{*}{ Freitag u. Vorfeiertage } & KR V & $373,86 \mathrm{DM}$ & $384,34 \mathrm{DM}$ & $22,60 \mathrm{DM}$ \\
\hline & KR Va & $384,06 \mathrm{DM}$ & $394,94 \mathrm{DM}$ & $23,23 \mathrm{DM}$ \\
\hline & KR VI & $398,89 \mathrm{DM}$ & $410,45 \mathrm{DM}$ & $24,14 \mathrm{DM}$ \\
\hline & KR VII & $411,13 \mathrm{DM}$ & $422,69 \mathrm{DM}$ & $24,86 \mathrm{DM}$ \\
\hline \multirow[t]{4}{*}{ Samstag } & KR V & $538,80 \mathrm{DM}$ & $553,90 \mathrm{DM}$ & $22,60 \mathrm{DM}$ \\
\hline & KR Va & $553,50 \mathrm{DM}$ & $569,18 \mathrm{DM}$ & 23,23 DM \\
\hline & KR VI & $574,87 \mathrm{DM}$ & $591,53 \mathrm{DM}$ & $24,14 \mathrm{DM}$ \\
\hline & KR VII & $592,51 \mathrm{DM}$ & $609,17 \mathrm{DM}$ & $24,86 \mathrm{DM}$ \\
\hline \multirow[t]{4}{*}{ Sonntag u. Feiertage } & KR V & $527,80 \mathrm{DM}$ & $324,99 \mathrm{DM}$ & $13,54 \mathrm{DM}$ \\
\hline & KR Va & $542,21 \mathrm{DM}$ & $333,96 \mathrm{DM}$ & $13,92 \mathrm{DM}$ \\
\hline & KR VI & $563,14 \mathrm{DM}$ & $347,07 \mathrm{DM}$ & $14,46 \mathrm{DM}$ \\
\hline & KR VII & $580,42 \mathrm{DM}$ & $357,42 \mathrm{DM}$ & $14,89 \mathrm{DM}$ \\
\hline
\end{tabular}

kleineren Fachbereiche äußerst fraglich. Des weiteren würden ungeahnte personelle Engpässe an Studientagen der Fachweiterbildung, bei Urlaub, Kur und Krankheit entstehen. Ein geregelter Tagesablauf kann dann aus meiner Sicht nicht mehr gewährleistet werden. Als weitere Folge greift dann auch die Regelung mit der Ruhepause zwischen 2 Diensten, die jetzt mit 11 Stunden festgelegt ist. Die Dienstbefreiung für geleistete Arbeitszeit an Samstagen, Sonn- und Feiertagen würde bei einer Schichtbesetzung an vorgenannten Tagen (4 Mitarbeiter Frühdienst, 4 Mitarbeiter Spät-
Wenn man nun diesen $\S 5$ und hier insbesondere den Absatz 3 nach dem Willen des Gesetzes durchführen muss, wie es in den Städtischen Kliniken Ffm.-Höchst der Fall ist, ist eine geordnete Personalsituation mit den Mitteln, die in Vertragsverhandlungen mit den Kassen erstritten werden, nicht mehr zu gewährleisten.

Nach dem Willen und Wünschen der Krankenkassen dürfen Fachkrankenschwestern und -pfleger für den Funktionsbereich in der Regel maximal 20 Jahre alt sein, Fachausbildung besitzen, 15 Jahre Berufserfahrung mitbringen und auf jeweilige Gehaltserhöhungen, die über KR V Stufe 1-2 liegen, für den Rest ihres Berufslebens verzichten. Dann kommt der Träger der Klinik mit dem Wunschbetrag von 76500,- inkl. Lohnnebenkosten aus.

Nur müsste den Politikern mittlerweile klar sein, dass man qualifiziertes Personal für dieses Entgelt nicht bekommt.

Durch die tarifliche Lohnerhöhung rückwirkend zum 1.1.1998 ist es bis heute im Rahmen der Verhandlungen mit den Kostenträgern noch strittig, wer, und aus welchem Etat die Lohnerhöhung aufgefangen wird.

Hieraus würde sich eine jährliche Mehrbelastung von 1125 DM ergeben.

\section{W. Werner}

Klinikoberpfleger der

Funktionsabteilungen gegen einen geordneten Tagesablau sprechen. Eine andere, wenn auch ebenso einschneidende Aufteilung der Dienstzeiten kann auch keine Gewähr für den reibungslosen Ablauf an den Werktagen bieten, in dem man die Gruppen halbiert und jeweils 2 Mitarbeiter als bereitschaftsdienstleistende je Schicht einbindet und 2 reinen Schichtdienst arbeiten lässt. Um den Dienstplan in ein solches Schema zu bringen, würden für den Bereich OP-Pflege ca. 60 bis 65 Mitarbeiter benötigt, für den Bereich der Anästhesie etwa 45 Pflegepersonen.
Städtische Kliniken

Frankfurt a. M.-Höchst

Gotenstraße 6-8

65929 Frankfurt 